\title{
EL CLIENTE COMO FACTOR PRIMORDIAL EN LA CADENA DE SUMINISTRO* Enfoque Servicio al Cliente
}

\author{
The customer as the primary factor in supply chain \\ DANiEla E. ALDANA**, ALEXANDRA MOSOS***, MARCELA VANEGAS****
}

Recibido: 20 de Octubre de 2015. Aceptado: 20 de Noviembre de 2015

DOI: http://dx.doi.org/10.21017/rimci.2016.v3.n5.a5

\begin{abstract}
Resumen
Nuestro equipo de trabajo en esta investigación hace énfasis en cómo se presta el servicio al cliente dentro de la logística que manejan las PYMES productoras y comercializadoras de productos cárnicos de bovino en la ciudad de Bogotá, esta información se obtuvo mediante cuestionarios tipo likert con cincuenta y dos preguntas acerca de las tres fases de servicio al cliente (pre transacción, transacción y post transacción). La finalidad es optimizar los procesos logísticos de la cadena de distribución del conglomerado, mediante la combinación de técnicas de optimización y las mejores prácticas de negocios proporcionando el nivel de servicio planificado.
\end{abstract}

Palabras clave: cadena de suministro, logística, Pymes, servicio al cliente.

\begin{abstract}
Our team in this research emphasizes how the service is delivered to the customer within that handle logistics producing and marketing of beef products in the city of Bogota, this information was obtained through questionnaires Likert about the three phases of customer service (pre transaction, transaction and post transaction). Finally it is to optimize the logistics processes of the supply chain cluster, by combining optimization techniques and best business practices to provide the level of service planned.
\end{abstract}

Keywords: supply chain, logistics, Pymes, customer service.

\section{INTRODUCCIÓN}

Este DOCUMENTO describirá la investigación partiendo de la caracterización de la gestión de pedidos que llevan a cabo las empresas del sector cárnico para la cumplimentación de pedidos que permite asegurar el máximo valor de la cadena de suministro, el servicio al cliente, la determinación de la estructura de la planificación y gestión del servicio al cliente como medida de actuación del sistema logístico que utilizan las empresas del sector cárnico para proporcionar en tiempo y lugar los productos y servicios a la cadena de distribución.

\footnotetext{
* Resultado del trabajo de semillero en el marco del proyecto: Identificación y caracterización logística del conglomerado Pymes de cárnicos de bovino en Bogotá D.C. del grupo de investigación O.C.A liderado por el Ingeniero Guillermo Montes Paniza del programa de Ingeniería Industrial de la Corporación Universitaria Republicana.

* Estudiante de décimo semestre de Ingeniería Industrial de la Corporación Universitaria Republicana. Correo electrónico: danielaaldana11 @hotmail.com

***Estudiante de décimo semestre de Ingeniería Industrial de la Corporación Universitaria Republicana. Correo electrónico: alexamosos@gmail.com

***** Estudiante de décimo semestre de Ingeniería Industrial de la Corporación Universitaria Republicana. Correo electrónico: marcelavanegas1 @gmail.com
} 


\section{Problema de Investigación}

Los cambios que actualmente se están produciendo en nuestro entorno colombiano por la internacionalización de la economía y la apertura de mercado dificulta la permanencia de empresas nacionales en el mercado nacional e internacional (Tomta y Chiatchoua) y están teniendo una poderosa influencia sobre las prácticas que están adoptando las industrias, incluyendo las pymes del sector cárnico, para gestionar las operaciones de negocio.

Actualmente la demanda del consumidor está centrada en una mayor exigencia de calidad y más servicios [1], tecnología según Attaran [2], precio, tiempo de entrega y calidad según Birendra [3], menor tolerancia al error [4], lo que se intensifica en el sector cárnico.

¿Cómo optimizar la cadena de distribución del conglomerado Pymes del sector cárnico de bovino de Bogotá, D. C., de tal forma que se satisfagan los requerimientos del cliente?

\section{Marco Conceptual}

\section{A. Servicio al Cliente}

El servicio al cliente representa a la vez la motivación y la consecuencia de todos los esfuerzos que la organización realice para cumplir con sus requerimientos. Se convierte entonces en una cultura organizacional; todas y cada una de las áreas y funciones de la empresa deben tener como objetivo cumplir las expectativas de los clientes por el grado de satisfacción de éstos determinará en gran medida los niveles de venta. Las funciones logísticas son las más comprometidas con este objetivo; son sus decisiones y acciones las que lo afectan en mayor grado.

El servicio al cliente se ha convertido también en un aspecto de gran relevancia y a la vez en una responsabilidad de las áreas de mercadeo. El profesor Jerome McCarthy introdujo las variables de producto, plaza, precio y promoción, en aquellos que llamó la mezcla de mercadeo [5]. Este concepto ha guiado las actividades de mercadeo a nivel mundial y hoy en día, se le incorpora el servicio (performance) como una quinta variable [6].
Para Karl Albrecht, una de las personas más conocedoras sobre el servicio al cliente, llegó el momento de pasar de la calidad total del producto a la calidad total del servicio. Las empresas que toman conciencia de la importancia de preocuparse por sus clientes, no lo han hecho únicamente para mejorar su imagen, lo hacen porque resulta definitivamente más rentable. Después de realizar algunas investigaciones, otro de los expertos en el servicio al cliente y que al igual a Albrecht que ha visitado Colombia, concluye que las empresas se han puesto a relacionar su participación en el mercado con la satisfacción de sus clientes. AT \& T, una de las empresas más importantes de comunicación, encontró que al aumentar la satisfacción de sus clientes en un $5 \%$, su participación en el mercado se incrementaba en casi la misma proporción. Sus investigaciones demuestran también que las empresas orientadas al cliente han crecido en promedio un $10 \%$ [7]. El servicio es fundamental, dada la competitividad de las compañías en tanto que, cuando el cliente adquiere un bien no sólo compra el producto o el servicio en sí, compra también una buena atención y un precio favorable.

Por su característica de intangible, el servicio no es definible en una sola idea, como pudiera hacerse con algún otro objeto o acción. Es por esto que el servicio al cliente lo definen las actividades que se realizan para lograr sus objetivos. Todos estos elementos, se dan al interior del marco delimitado por tres parámetros a saber: tiempo de entrega, confiabilidad en la información y disponibilidad de producto.

El enmarcar el funcionamiento del servicio al cliente en estas tres dimensiones, sugiere que ninguna puede estar ausente a lo largo del proceso; bastará con que falle sólo una de estas para que el flujo de la cadena de abastecimiento se vea afectado y los objetivos no se cumplan en su totalidad [6].

\section{B. Elementos del Servicio al Cliente}

El proceso de análisis de dependencias funcionales (DF) fue realizado para transformar el modelo de datos en un esquema completo, que además ofrece la implantación de las restricciones de diseño, con la finalidad de que el RDBMS asumiera la carga de asegurar la consistencia de la base de datos. Ver figura 1. 


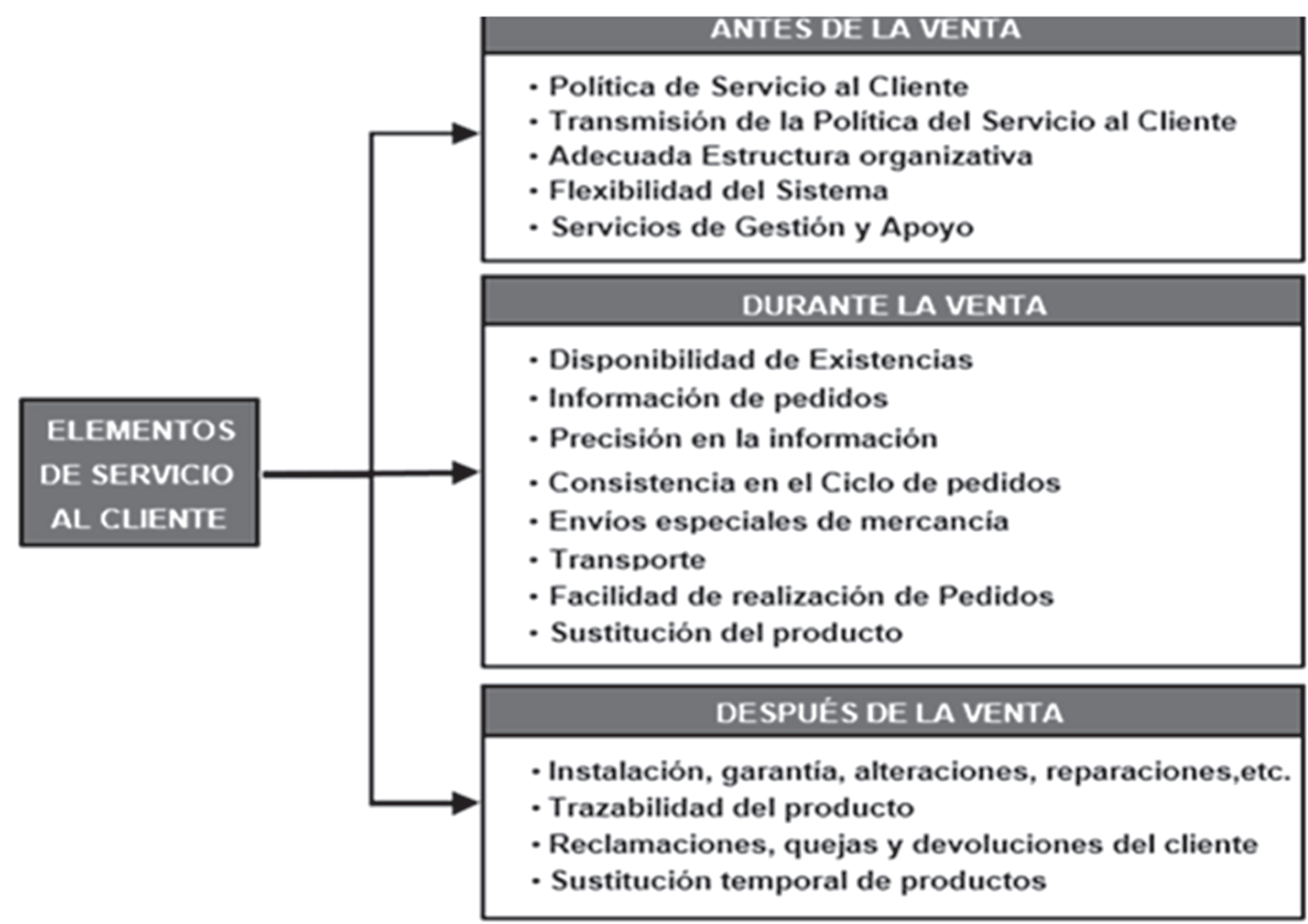

Fig. 1. Elementos del servicio al cliente. Fuente: Pilot, 2002 [1]

De acuerdo con el manual de Pilot, el servicio al cliente se dividen en los siguientes elementos: pre transacción, transacción y post transacción.

En la pre transacción se encuentran las políticas establecidas por la organización enfocadas al servicio al cliente, la flexibilidad como prioridad competitiva del sistema, servicios técnicos tanto de gestión como de apoyo, entre otros.

El servicio al cliente es un aspecto fundamental de la cadena de suministro, por lo tanto es importante que sea valorado, para cumplir con esto, se presenta una desventaja, ya que solo puede ser evaluado después de que haya concluido y no mientras es ejecutado, por esto, la probabilidad de corregir los errores antes de que finalice el proceso tiende a ser nula.

De allí surge la necesidad de establecer políticas de servicio al cliente y así mitigar las falencias antes de que conviertan en errores. La política de servicio al cliente según Tupperware Colombia, se encarga de comunicar a los clientes lo que pueden esperar de la organización en términos de servicio, estas deben dejar en claro la misión, visión, justificación, objetivos, alcance, políticas específicas en procesos detallados como recepción de solicitudes, quejas, reclamos, peticiones y sugerencias. Dando a conocer el procedimiento a seguir en recepción de pedidos, anulación de pedidos, premios, faltantes, sobrantes, garantías, facturación, cartera, al igual que el horario de atención y seguimiento de solicitudes.

En conclusión, la política de servicio al cliente es el contrato entre la organización de la logística y el cliente; en ella se definen las metas y objetivos del servicio, establece los requisitos de servicio para cada proceso logístico, incluyendo la gestión de inventarios, suministros, transporte y almacenamiento. Es la base de la planificación general de la logística, refleja la cultura y madurez logística de la organización.

Los elementos de la transacción son aquellos que tienen como finalidad entregar el producto al cliente, entre ellos están los niveles de inventarios, 
información sobre pedidos y habilidad para manejar pedidos atrasados, elementos del ciclo de pedido, formas de transporte, tiempo de entrega, trasbordo, precisión del sistema, conveniencias del pedido, sustitución del producto, envíos especiales, tiempo (lead time) [8].

En la post transacción están los elementos que se demandan para sostener el producto en el campo y proteger a los clientes de productos defectuosos; trazabilidad del producto; reclamaciones, quejas y devoluciones del cliente; empacado del producto; instalación, garantía, alteraciones; sustitución temporal de producto durante mantenimiento [8].

\section{Calidad en el servicio al cliente}

Hoy día, la atención al cliente es una actividad desarrollada por las organizaciones con orientación a satisfacer las necesidades de sus clientes, logrando así incrementar su productividad y ser competitiva.

El cliente es el protagonista principal y el factor más importante en el juego de los negocios. Si se examina los enfoques modernos que se dan en las diferentes organizaciones, se puede apreciar que para tener éxito en la atención al cliente, existen varios elementos que la empresa debe tener en cuenta, como son el liderazgo, eficiencia en sus operaciones, capital humano, cultura organizacional y esta última debe estar bien definida para que sus integrantes conozcan la misión y visión de la empresa, de esta forma los trabajadores al sentirse identificados y parte de la misma, estarán motivados a realizar sus actividades, con calidad y esto influye positivamente en la atención a sus clientes [9].

Teniendo en cuenta lo anterior se puede tomar como ejemplo la metodología y/o estrategias por el cual el Fondo Nacional de Garantías rige su política de servicio al cliente de acuerdo a las estrategias de servicio que alineadas con las políticas de servicio se establecen estrategias internas y externas que permiten satisfacer las necesidades de sus clientes actuales y la vinculación de futuros clientes.

\section{A. Cultura del Servicio en el FNG}

Significa responder de manera oportuna, con calidad y respeto, todas las solicitudes recibidas de los clientes internos y externos del FNG. Los comportamientos facilitadores para desarrollar la Cultura de Servicio del FNG, que deben desarrollar los funcionarios del FNG, son los siguientes[10]: (figura 2).

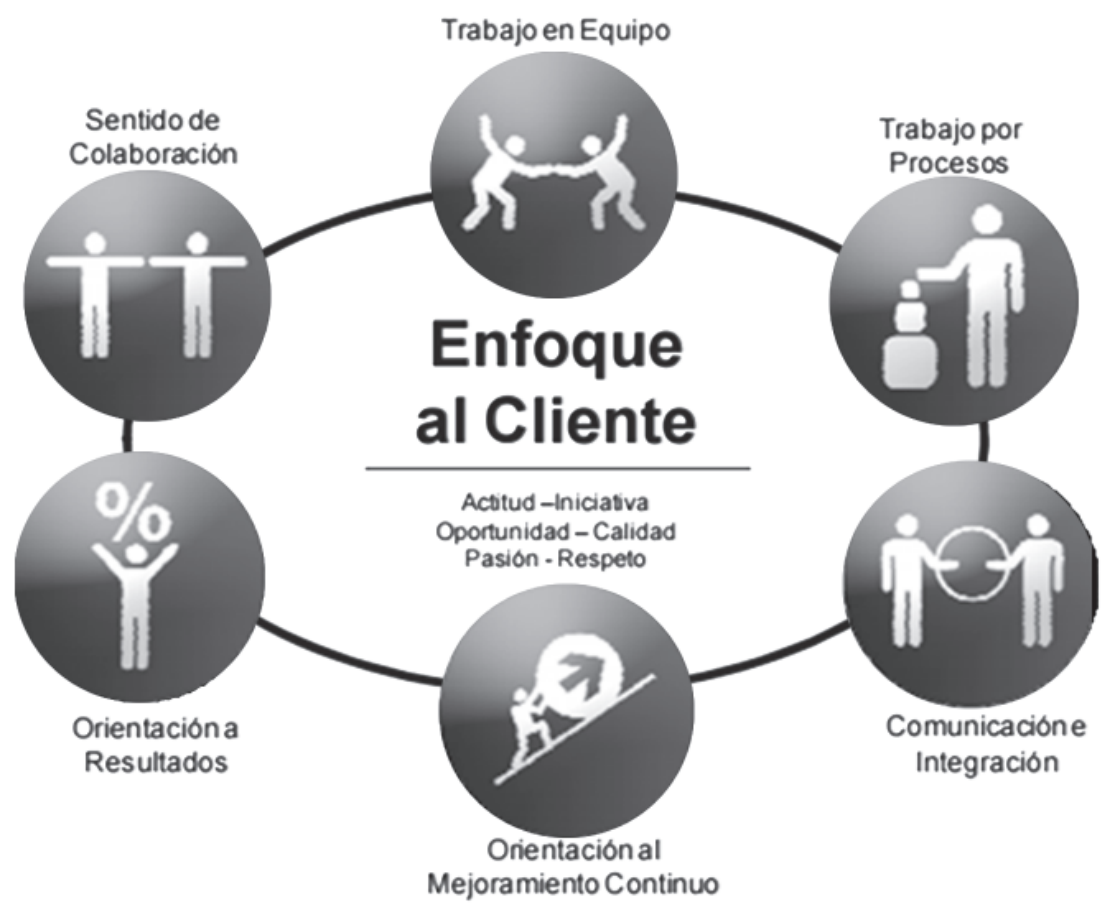

Fig. 2. Elementos del servicio al cliente.Fuente: Fondo Nacional de Garantías [10] 
DANIELA E. ALDANA, ALEXANDRA MOSOS, MARCELA VANEGAS

1) Sentido de Colaboración: Ayudar y servir de manera espontánea a los demás, hasta en los pequeños detalles.

2) Trabajo en Equipo: Compartir la misma dirección y el sentido del FNG, permite llegar más rápido y fácilmente al destino, porque ayudándonos entre nosotros los logros son mejores.

3) Trabajo por Procesos: Permanecer en sintonía $\mathrm{y}$ unidos junto a aquellos que se dirigen en nuestra misma dirección, el esfuerzo será menor.

4) Comunicación e integración entre Áreas: Reunir habilidades y capacidades, combinar dones, talentos y recursos.

5) Orientación al Mejoramiento Continuo: Es la capacidad para enfocar nuestra actuación en una práctica permanente para superar las expectativas, e innovar para optimizar y hacer más eficientes los procesos.

\section{Metodología}

El desarrollo del presente artículo parte de la investigación efectuada por los integrantes del semillero «Identificación y caracterización logística del conglomerado pymes de cárnicos de bovino en Bogotá, D.C.» guiado por el Ingeniero Guillermo Enrique Montes, que tiene como finalidad proponer estrategias para la planificación y gestión del servicio al cliente que permitan proporcionar en tiempo y lugar al menor costo los productos y servicios ofertados al cliente. Además, señalar métodos que permitan tomar decisiones óptimas en cuanto a la selección del modo de transporte, diseño de rutas, programación de vehículos y consolidación de envíos. Adicionalmente, resaltar las estrategias para optimizar la gestión de pedidos que incide en la cumplimentación de pedidos que asegura el máximo valor de la cadena de suministro y el servicio al cliente.

\section{Resultados}

Una adecuada descripción estructural de la logística empresarial de las pymes del sector cárnicos, promoverá bases sólidas para adelantar posteriores investigaciones en diferentes sectores y/o líneas de producción. La caracterización de la estructura logística empresarial es un mecanismo válido que genera valor económico y beneficios para todos los actores que componen el conglomerado de las Pymes del sector cárnicos de Bogotá D.C. Una adecuada concepción de la logística empresarial, permite cambiar a los empresarios pymes el paradigma empresarial de manejar estructuras en términos de silos funcionales a procesos internacionales.

Una gestión efectiva de la logística en el sector manufacturero de cárnicos en pymes de Bogotá D. C., permite generar la interacción e integración para optimizar los procesos desde el sector primario hasta el consumidor final para obtener ventajas competitivas.

El equipo de trabajo al aplicar los cuestionarios en las diferentes pymes observó que muchas de ellas no tienen una política del servicio al cliente escrita aunque de una u otra forma esta divulgada, además no aplican indicadores de gestión para medir su nivel de servicio, simplemente se basan en datos históricos de ventas y lo que expresa el cliente al ser culminada la transacción. Se puede decir que todos tienen el objetivo claro que es el de satisfacer las necesidades de sus clientes brindando productos de calidad, creando confianza y seguridad, con la finalidad de fidelizar al consumidor y así crear un vínculo, además todas estas pequeñas y medianas empresas buscan la constante mejora, algunas de ellas por medio de capacitaciones, ya que las que fueron sometidas al cuestionario se encuentran en clusters, es decir, su entorno es competitivamente fuerte.

\section{CONCLUSIÓN}

Las empresas pasan por diferentes y abundantes desafíos día a día, para ellos no es simplemente tener una buena actitud y hacerlo bien, es ser excelentes en su campo. Se vive en un ambiente en donde crece la competitividad el cual obliga a las pymes a ser más eficientes teniendo procesos claros y estrategias puntuales para poder progresar. Aquí el cliente desempeña un papel con autoridad de negociación además de ser jurado en la victoria o fracaso de las empresas. Al realizar esta 
investigación se confirmó que un producto es de calidad cuando pasa por el gran título «cadena de suministro» y que el proceso no termina cuando se comercializa si no cuando aquel cliente da por hecho que su necesidad ha sido suplida.

\section{REFERENCIAS}

[1] Programa-de-Innovación-Logística, «Pilot Sistemas de Información y Herramientas en SCM». Zaragoza: ed. Instituto Aragonés de Fomento, 2002.

[2] Attaran, M. and Attaran S., «Collaborative supply chain management. The most promising practice for building efficient and sustainable supply chains» Business Process Management Journal., vol. 13, no. 3, pp. 390-404, 2007.

[3] Birendra, M., Srinivasan, R. and Xiaohang, Y., "Information sharing in supply chains: incentives for information distortion,» IEEE Transactions, vol. 39, no. 9, pp. 863-877, 2007.
[4] Cámara de Comercio de Medellín para Antioquia, «Perspectivas de la gestión logística,» Herramientas empresariales, 2014 . .

[5] J. McCarthy, W. Perreault, MARKETING: UN ENFOQUE GLOBAL, 13th ed. México: McGraw-Hill Interamericana de México.

[6] M. Gómez, G. Aragón, M. Moschner, «Servicio Logístico al Cliente Generador de Ventajas Competitivas Ingeniería y competitividad» Revista científica y tecnológica, vol. 1, no. 2, 1998.

[7] El Tiempo, «El cliente es lo de menos» 18-Feb-1996.

[8] R. H. Ballou and C. Mendoza, Logística?: administración de la cadena de suministro. México: Pearson/ Educación, 2004.

[9] Observatorio Laboral, «El capital humano en la atención al cliente y la calidad de servicio» Revista Venezolana. Universidad de Carabobo, vol. 4, no. 8, pp. 25-35, 2011.

[10] Fondo Nacional de Garantías S.A, «Documento servicio al cliente» SGD. 